LA-UR-99-4171

\title{
Analysis of Dislocation Mechanism for Melting of Elements
}

\author{
Leonid Burakovsky* and Dean L. Preston ${ }^{\dagger}$ \\ Los Alamos National Laboratory \\ Los Alamos, NM 87545, USA
}

\begin{abstract}
The melting of elemental solids is modelled as a dislocation-mediated transition on a lattice. Statistical mechanics of linear defects is used to obtain a new relation between melting temperature, crystal structure, atomic volume, and shear modulus that is accurate to $17 \%$ for at least half of the Periodic Table.
\end{abstract}

Key words: dislocation, lattice, phase transition, melting

PACS: 11.10.Lm, 11.27.+d, 61.72.Bb, 64.70.Dv, 64.90.+b

Phase transitions in many physical systems can be understood in terms of an abrupt proliferation in the number density of line-like structures. Onsager [1] suggested in 1949 that the $\lambda$-transition in liquid ${ }^{4} \mathrm{He}$ could be described in terms of the unbinding of vortex lines. The modeling of melting as a dislocation-driven phase transition was advocated by Shockley [2]. Edwards and Warner [3] later constructed a theoretical model for dislocation-mediated melting and applied it to the melting of iron. More recently, the elementary defect theory of melting of refs. [1-3] has been developed to a higher degree of sophistication in the textbook by Kleinert [4. Other examples include: (i) the superconducting/normal conducting transition in bulk superconductors [5], where the line-like structures are magnetic flux lines, (ii) the deconfinement transition in QCD [6], where these structures are color flux tubes, (iii) the condensation of synthetic macromolecules [7] and the viral DNA helicity transition [8], where the line-like structures are macromolecules, and (iv) the glass transition [9], where linear defects are $Z_{2}$ disclinations.

\footnotetext{
${ }^{*}$ E-mail: BURAKOV@T5.LANL.GOV

${ }^{\dagger}$ E-mail: DEAN@LANL.GOV
} 
In this paper we will show that linear defects, namely dislocations, drive the melting transition and lead to a simple relation, a new dislocation-mediated melting law, between melting temperature, crystal structure and elasticity data. The relation holds for a surprisingly large number of elements, and should also apply to alloys and compounds, such as salts.

Although the energy per unit length, $\sigma$, of a dislocation can be very large, this energy can always be compensated at sufficiently high temperatures by the large entropy of linelike structures. In order to see this, consider an ensemble of static non-interacting linear defects with energy per unit length $\sigma$ on a $d=3$ dimensional simple cubic lattice of volume $V$ and lattice constant $a$. The grand canonical partition function for such an ensemble at temperature $T \equiv 1 / \beta\left(k_{B}=1\right)$ is $Z=\exp \left\{Z_{1}\right\}$, where

$$
Z_{1}=\sum_{\text {strings }} e^{-\beta E}=\sum_{L} n(L) e^{-\beta \sigma L}
$$

is the canonical partition function for a single static linear defect, and $n(L)$ is the number of configurations of a defect of length $L$. For simplicity, we require the defects to lie along the links of the lattice. Then, for $L \gg a$ [10]

$$
n(L) \simeq \frac{V}{a^{3}}\left(\frac{L}{a}\right)^{-q-1}\left(z^{\prime}\right)^{L / a}
$$

where $z^{\prime}$ is the number of possible directions that a defect segment can take from a given lattice point. $z^{\prime}=z-1$ if backtracking is not allowed, and $z^{\prime}=z$ otherwise, where $z$ is the coordination number; $z=2 d$ for a simple cubic lattice in $d$ dimensions. The parameter $q$ accounts for the topology of the defect network: $q=-1$ for non-interacting open defects, $q=d / 2$ for non-interacting closed defects, and $q=7 / 4$ for non-self-intersecting defects at low defect densities in 3 dimensions [10]. Thus,

$$
Z_{1} \simeq \frac{V}{a^{3}} \sum_{L}\left(\frac{L}{a}\right)^{-q-1} e^{-\beta \sigma_{\mathrm{eff}} L},
$$

where

$$
\sigma_{\text {eff }} \equiv \sigma-\frac{T \ln z^{\prime}}{a}
$$

is the effective energy cost to create unit length of a defect at temperature $T$. At the critical temperature $T_{c r} \equiv \sigma a / \ln z^{\prime}$, there is no energy cost to produce a defect, and therefore defects proliferate as $T_{c r}$ is approached from below. At temperatures above $T_{c r}$, the divergence of $Z_{1}$ signals the breakdown of the underlying theory, and the system enters a new phase. Hence, the temperature $T_{c r}$ corresponds to a phase transition (the particle physics analog of which is the so-called Hagedorn transition) in which linear defects are copiously produced. We thus consider the melting of elements to be a dislocation-mediated transition, and equate the melting temperature, $T_{m}$, to $T_{c r}$.

Before proceeding further, however, we briefly address the question of the order of the melting transition in this approach. With a constant dislocation energy per unit length, the phase transition discussed above is second order. However, in view of Eq. (5) below, the dislocation self-energy is expected to be a function of dislocation density: it decreases with dislocation density because of screening. Although the $\rho$ dependence of the 
self-energy does not follow rigorously from theory, estimates made in [11, 12] show that such dependence would result in $-\rho \ln \rho$ dependence of the free energy of the dislocation ensemble on dislocation density, and lead to a first order melting transition that occurs when the free energy of the crystal with a sufficiently high dislocation density equals the free energy of the dislocation-free crystal. Recently, this mechanism of a first-order phase transition in a system of dislocations has been applied to vortex line lattices [13].

An alternative melting mechanism is discussed by Kleinert in his textbook [4], where it is suggested that the interplay of dislocations and disclinations is essential for explaining both the strong first-order nature of melting and the transition entropy. In fact, our framework describes dislocations, which do not exist in liquids. Moreover, although a pure dislocation melting can be first order, as discussed above, it can only explain a transition to a translationally disordered solid, not a rotationally disordered liquid. Thus, disclinations certainly play a role in the melting process. If a dislocation is viewed as a disclination dipole [4], the transition to a translationally disordered solid corresponds to the proliferation of the disclination dipoles. The translationally disordered solid may then undergo a Kosterlitz-Thouless-type vortex-unbinding transition [14 to a phase of free disclinations, viz., a liquid. In this paper we do not discuss the full defect model of melting, as a version of it is presently available [4. Instead, we wish to see how close we can estimate transition temperatures on the basis of the energy-entropy argument of dislocation lines alone, $\sigma_{\text {eff }}=0$ in Eq. (4). We hope thereby that the possible shift in the melting temperatures caused by the disclinations is small enough to be negligible.

In order to apply Eq. (4) to get the melting temperature, we take the dislocation energy per unit length to be that of a dislocation in a complex array or tangle of other dislocations. In that case the stress field of a given dislocation beyond the mean interdislocation spacing, $R$, is largely cancelled out by the stress fields of the other dislocations in the complex array. It then follows that 15]

$$
\sigma=\kappa \frac{G b^{2}}{4 \pi} \ln \left(\alpha \frac{R}{b}\right)=\kappa \frac{G b^{2}}{4 \pi} \ln \left(\frac{\alpha}{b \sqrt{\rho}}\right) .
$$

Here, $\kappa$ is 1 for a screw dislocation, and is equal to $(1-\nu)^{-1} \approx 3 / 2$ for an edge dislocation, $\nu$ being the Poisson ratio. Edge dislocations are energetically suppressed. Also, $G$ is the shear modulus, $b$ is the average Burgers vector magnitude, and $\alpha$ is a constant of order unity. In the second half of this equation we have taken the distance $R$ to be approximately equal to $1 / \sqrt{\rho}$, where $\rho$ is the dislocation density. Eq. (5) accounts for both the linear elastic and core energies.

Using the above expression for the dislocation energy per unit length, the formula for the melting temperature of a simple cubic lattice becomes

$$
T_{m}=\frac{G a b^{2}}{4 \pi \delta \ln z^{\prime}}, \quad \delta^{-1} \equiv \kappa \ln \left(\frac{\alpha}{b \sqrt{\rho\left(T_{m}\right)}}\right) .
$$

The derivation of the corresponding expressions for the melting temperatures of bodycentered cubic (bcc), face-centered cubic (fcc), hexagonal close-packed (hcp), ..., elements within the framework of statistical mechanics of line-like defects would involve greater geometric complexity. However, we expect that $T_{m}$ for any lattice is given by the expression (6) with a replaced by the cube root of the volume per atom, and a structure factor of 
order one. Also, $z$ retains its meaning as the coordination number, that is, the number of nearest-neighbor atoms in a crystal.

We assume that no backtracking is allowed for dislocations, since each backtracking would result in a divergence in the linear elastic interaction energy between the overlapping segments. We therefore choose $z^{\prime}=z-1$. The coordination numbers for the elements considered in our analysis are $z=6$ for the rhombohedral (rhomb) lattice, $z=8$ for the bcc lattice, and $z=12$ for the fcc, hcp, and double hcp (dhcp) lattices. Since the magnitude of the Burgers vector is approximately equal to the interatomic distance in all of these lattices, $b$ can also be replaced by the cube root of the volume per atom and a structure factor of order one. After absorbing factors of order one into $\kappa$, we obtain our formula for the melting temperature of the elements:

$$
\frac{G v}{4 \pi N T_{m} \ln (z-1)}=\delta
$$

where $v$ is the unit cell volume and $N$ is the number of atoms per unit cell. The approximations and assumptions leading to this formula are justified a posteriori by its accuracy. We will now evaluate the left-hand-side of Eq. (7) for two-thirds of the elements of the Periodic Table using the measured values of $G, T_{m}$ and lattice constants.

Both $G$ and $v$ in Eq. (7) are in general temperature-dependent quantities, and so, as with $\rho\left(T_{m}\right)$, their values to be used in $(7)$ should be those at $T=T_{m}$, not the measured values at room temperature. However, measurements of $G$ and $v$ above $0.8 T_{m}$ are available only for $\mathrm{Na}, \mathrm{K}, \mathrm{Al}, \mathrm{Cd}$ and $\mathrm{Zn}$ [16]. Consequently, we used the values of $G$ and $v$ at room temperature. The decrease in $G$ with temperature is partially compensated by the increase of the volume of the unit cell. Between room temperature and $T_{m}, G$ drops typically by about 20\% [17], while the volume increase is of order $5 \%$ [18], so we expect our values of $\delta$ to be $\sim 15 \%$ high.

Elements excluded from our analysis are those that exist in different allotropes, e.g., B, Bi, C, H, He, Li, P, S, Si; those that have more than 3 polymorphic phases, e.g., Mn, $\mathrm{Pu}$; and those for which we could not find the values of one or more of the parameters to be used in the analysis. For example, we could not find the values of $G$ for At, $\mathrm{Br}, \mathrm{Cl}, \mathrm{F}$, I, N, O, Rn, although the corresponding lattice constants are known.

For the elements that are included in our analysis, the values of $a$ are taken from [18], except for Fr (estimated using the formulas of ref. [19). The values of both $T_{m}$ and $G$ are mostly taken from [20]. We also calculate some values of $G$ from those of the single crystal elastic constants [21] using known relations [22]. For $\mathrm{Pb}$ and $\mathrm{Tl}$, the deviation of the calculated value from the experimental one [20] is larger than $50 \%$, so we use the calculated value based on more accurate data [21]. In other cases, the deviation of our calculated values from those of [20] is $39 \%$ for Na, and less than $20 \%$ for all the remaining materials, so that we use the experimental values. The values of $G$ for $\mathrm{Ar}, \mathrm{Kr}, \mathrm{Ne}$ and $\mathrm{Xe}$ are also calculated. The values of $T_{m}$ for $\mathrm{Ar}, \mathrm{Kr}, \mathrm{Ne}$ and Xe are from 18.

The volumes of the unit cells we use are $v=a^{3}$ for bcc and fcc, $v=a^{2} c \sqrt{3} / 2$ for hcp and dhcp, and $v=a^{3} \sin \alpha \sin \beta, \cos \beta=\cos \alpha / \cos (\alpha / 2)$ for rhombohedral lattices. For polymorphic elements, the conversion temperatures are taken from 18. For all such elements except Sc, lattice constants are measured in all solid phases. Eq. (7) can be consistently applied to these solid phases by extrapolating the values of $G$ to the phase 
boundaries, using the empirical relation [17

$$
G(T) \simeq G(0)\left(1-\gamma \frac{T}{T_{m}}\right)
$$

with $\gamma=0.23 \pm 0.08$, which was calculated by fitting to data on thirteen metals. The shear-modulus data on these elements span a sufficiently broad range of temperatures, specifically $T=0$ to $T / T_{m} \gtrsim 0.4$, that $\gamma$ can be accurately determined. For polymorphic elements, except for Sc, we calculate $\delta$ in the crystal structure at melt (always bcc except for Co). Interestingly, $\delta$ generally changes very little even when it is evaluated in the lower-temperature solid phases.

Our results for $\delta$, i.e., for the ratio given in Eq. (7), are shown in Fig. 1.

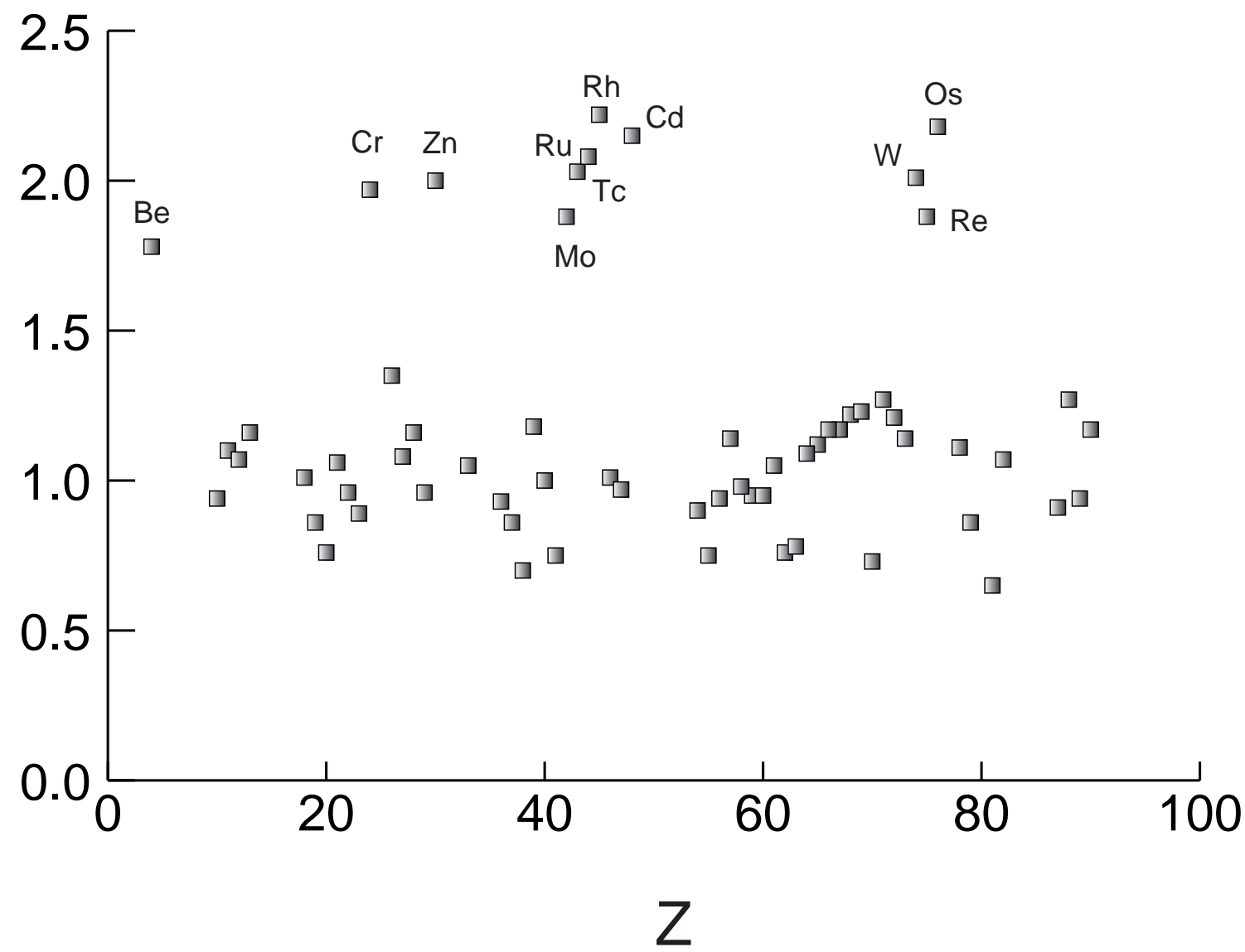

Fig. 1. Values of $\delta=G v_{W S} / 4 \pi T_{m} \ln (z-1)$ from experimental data for 62 elements.

The 11 elements that form an outlying group in Fig. 1 are explicitly labelled. We refer to these 11 elements, for which $\delta=2.02 \pm 0.13$, as anomalous, and the remaining 51 elements, for all of which $\delta=1.01 \pm 0.17$, as regular. We find $\delta=1.19 \pm 0.42$ for the entire set of 62 elements. 
In Table 1 we make some predictions for the shear moduli of the actinides Am, Bk, $\mathrm{Cm}$ and Es, assuming that they all are regular $(\delta=1.0 \pm 0.2)$. The melting temperatures, and the polymorphic conversion temperatures of $\mathrm{Am}, \mathrm{Bk}$ and $\mathrm{Cm}$ (second column) are taken from 23.

\begin{tabular}{|c|c|c||c|c|c||c|}
\hline element & structure & $N$ & $a, \AA$ & $c, \AA$ & $T_{m}, \mathrm{~K}$ & $G, \mathrm{GPa}$ \\
\hline \hline $\mathrm{Am}$ & dhcp/1044/fcc/1347/bcc & $\mathbf{4} / 4 / 2$ & 3.468 & 11.240 & $1447 \pm 2$ & $20.6 \pm 4.1$ \\
$\mathrm{Am}$ & dhcp/1044/fcc/1347/bcc & $4 / \mathbf{4} / 2$ & 4.894 & & $1447 \pm 2$ & $21.9 \pm 4.4$ \\
$\mathrm{Bk}$ & $\mathbf{d h c p} / 1250 /$ fcc & $\mathbf{4} / 4$ & 3.416 & 11.069 & 1323 & $19.7 \pm 3.9$ \\
$\mathrm{Bk}$ & $\mathrm{dhcp} / 1250 /$ fcc & $4 / \mathbf{4}$ & 4.997 & & 1323 & $22.5 \pm 4.5$ \\
$\mathrm{Cm}$ & $\mathbf{d h c p} / 1550 /$ fcc & $\mathbf{4} / 4$ & 3.500 & 11.134 & 1618 & $22.8 \pm 4.6$ \\
$\mathrm{Es}$ & $\mathrm{fcc}$ & 4 & 5.746 & & 1133 & $9.9 \pm 2.0$ \\
\hline
\end{tabular}

Table 1. Predictions for the values of $G$ for the actinides Am, Bk, Cm and Es. Boldface indicates the structure used for the calculation of $G$ with the help of Eq. (7).

For the regular 51 elements with $\delta \simeq 1$, it follows from Eq. (6) that the dislocation density at melt is

$$
\rho\left(T_{m}\right) \simeq(e b)^{-2} \approx(2.7 b)^{-2}
$$

We have set $\alpha=\kappa=1$. In the case of $\mathrm{Cu}, b \approx 2.55 \AA$ [24, so that $\rho\left(T_{m}\right) \simeq 2 \cdot 10^{14} \mathrm{~cm}^{-2}$, which corresponds to a mean separation between dislocations at melt of about $7 \AA$. For comparison, consider Cotterill's estimate [24] of the threshold dislocation density above which the atoms in a crystal rearrange into an amorphous state. Under the assumption that rearrangement does not occur until the amount of slip plane between $b$ (approximately equal to the core radius) and $R$ is insufficient to withstand the core stress, he derived the inequality $G(R-2 b) b \geq 4 \pi E_{\text {core }}$, which must be saturated at the threshold dislocation density, $\rho^{t h}$. Using the core energy per unit length $E_{\text {core }}=G b^{2} / 4 \pi$ [15] (Cotterill used a value proportional to the latent heat of fusion) we obtain $\rho^{t h} \simeq(3 b)^{-2}$, which agrees with our linear defect model estimate (9). We have therefore found that dislocation density at melt is close to its limiting value as estimated by Cotterill.

Poirier and Price [25] identified Zn and Cd, the two anomalous elements with relatively low melting temperatures, as irregular, but could find no explanation for their behavior, besides their non-ideal hcp structure with a large $c / a$ ratio. The irregular properties of $\mathrm{Cr}, \mathrm{Mo}, \mathrm{W}$, and Be, Cd, Zn were also noted in [18].

The best known melting rule is that of Lindemann, which assumes that all elements melt when the atomic vibrational amplitude is a fixed fraction, $\approx 1 / 8$, of the interatomic distance. Wallace [26] calculates the value of $\ln L$ for 28 elements which he identifies as regular, where $L$ is the Lindemann parameter. Based on this value, we calculate the precision of the Lindemann rule for these 28 elements to be $12 \%$. Wallace also calculates the value of $\ln L$ for 8 elements which he identifies as irregular (which consist of Cr, Mo, W, 3 of our 11 anomalous elements, and 5 additional elements which are absent from our analysis of 62 elements). Based on Wallace's listing of $\ln L$, we calculate the precision of the Lindemann rule to be $35 \%$ for all 36 elements of ref. [26]. Our linear defect theoretical formula (7) is accurate to $17 \%$ for over at least half of the Periodic Table, and to $35 \%$ for the entire set of 62 elements that we analyzed. It is therefore not less accurate than 
the Lindemann rule (taking into account a fewer number of regular elements in Wallace's analysis).

We now offer a possible explanation for anomalous melting. The translationally disordered solid phase, which precedes the liquid phase, is represented in our model by a scale-invariant distribution of dislocation loops, namely $n(L) \propto L^{-q-1}$. We suspect that for the anomalous metals this unique multi-defect state is a poor representation of the solid at the melting point, because they actually melt before losing all of their crystalline structure. Hence, we expect the anomalous metals to have relatively large (negative) liquid correlation entropies, $S_{c}^{\text {liq }}$. Wallace [26] has identified $\mathrm{Cr}$, Mo and $\mathrm{W}$, all anomalous elements, as having values of $S_{c}^{\text {liq }}$ outside the normal range. We also expect the melting temperatures of the anomalous elements to lie below the values predicted by our linear defect model with $\delta=1$ (normal element). This is indeed the case - the melting temperatures of all anomalous elements are suppressed $(\delta>1)$ but never elevated $(\delta<1)$. Anomalous melting can be accounted for in our model by assuming that it occurs when

$\sigma_{\text {eff }} \simeq G b^{2} /(8 \pi)=O(0.1) \mathrm{eV} \AA^{-1}$. Of course, other explanations for anomalous melting can be suggested as well.

To conclude, we have used the methods of statistical mechanics of linear defects to derive a new melting rule. It involves only the crystal structure, atomic volume, and shear modulus, but despite its simplicity it is accurate to $17 \%$ for at least half of the Periodic Table. A preliminary study of our melting rule as applied to alloys and compounds indicates an accuracy comparable to that for the elements. These results will be presented in a longer paper where we will also apply our melting rule to the calculation of melting curves.

\section{Acknowledgements}

We wish to thank R.R. Silbar and T. Goldman for very valuable discussions during the preparation of this work. One of us (D.L.P.) would like to express his gratitude to D.C. Wallace for several very informative discussions on melting.

\section{References}

[1] L. Onsager, Nuovo Cim. Suppl. 6 (1949) 249, Ann. N.Y. Acad. Sci. 51 (1949) 627

[2] W. Shockley, in L'Etat Solide, Proceedings of Neuvienne-Consail de Physique, Brussels, 1952, Ed. R. Stoops, (Solvay Institut de Physique, Brussels, Belgium)

[3] S.F. Edwards and M. Warner, Phil. Mag. A 40 (1979) 257

[4] H. Kleinert, Gauge Fields in Condensed Matter, (World Scientific, Singapore, 1989), Vol. II

[5] D.J. Amit, Field Theory, the Renormalization Group and Critical Phenomena, (McGraw-Hill, New York, 1978)

[6] A. Patel, Nucl. Phys. B 243 (1984) 411 
[7] F.W. Wiegel, in Phase Transitions and Critical Phenomena, Eds. C. Domb and J. Lebowitz, (Academic Press, New York, 1973), Vol. 7

[8] R.M. Wartell and E.W. Montroll, Adv. Chem. Phys. 22 (1972) 129

[9] N. Rivier and D.M. Duffy, J. Phys. C 15 (1982) 2867

[10] E. Copeland, D. Haws, S. Holbraad and R. Rivers, Physica A 158 (1989) 460, Phys. Lett. B 236 (1990) 49

[11] S. Mizushima, J. Phys. Soc. Japan 15 (1960) 70

[12] R.M.J. Cotterill, J. Crystal Growth 48 (1980) 582

[13] J. Kierfeld and V. Vinokur, Dislocations and the critical endpoint of the melting line of vortex line lattices, cond-mat/9909190

[14] J.M. Kosterlitz and D.J. Thouless, J. Phys. C 6 (1973) 1181, in Progress in LowTemperature Physics, Vol. VII-B, Ed. D.F. Brewer, (North-Holland, Amsterdam, 1978), p. 373. The $3 D$ Kosterlitz-Thouless transition is discussed in N.K. Kultanov and Yu.E. Lozovik, Solid State Comm. 88 (1993) 645, Physica Scripta 56 (1997) 129

[15] J.P. Hirth and J. Lothe, Theory of Dislocations, 2nd ed., (Krieger Publishing, Malabar, FL, 1992)

[16] G. Simmons and H. Wang, Single Crystal Elastic Constants and Calculated Aggregate Properties, 2nd ed., (MIT Press, Cambridge, MA, 1971)

[17] D.L. Preston and D.C. Wallace, Solid State Comm. 81 (1992) 277

[18] Landolt-Börnstein Numerical Data and Functional Relationships in Science and Technology, New Series, Eds. K.-H. Hellwege and O. Madelung, Group III: Crystal and Solid State Physics, (Springer-Verlag, Berlin), Vol. 7 (1971), Vol. 14 (1988), Vol. 25 (1991)

[19] R.D. Shannon, Acta Crystallogr. A 32 (1976) 751

[20] K.A. Gschneidner, Jr., in Solid State Physics, Advances in Research and Applications, Eds. F. Seitz and D. Turnbull, (Academic Press, New York, 1965), Vol. 16, p. 275

[21] Landolt-Börnstein Numerical Data and Functional Relationships in Science and Technology, New Series, Eds. K.-H. Hellwege and O. Madelung, Group III: Crystal and Solid State Physics, (Springer-Verlag, Berlin), Vol. 11 (1979), Vol. 18 (1984)

[22] P. Sisodia and M.P. Verma, J. Phys. Chem. Solids 50 (1989) 223

[23] J.W. Ward, P.D. Kleinschmidt and D.E. Peterson, in Handbook on the Physics and Chemistry of the Actinides, Eds. A.J. Freeman and C. Keller, (North-Holland, Amsterdam, 1986), Vol. 4, Chap. 7

[24] R.M.J. Cotterill, Phys. Lett. A 60 (1977) 61 
[25] J.P. Poirier and G.D. Price, Phys. Earth Planet. Inter. 69 (1992) 153

[26] D.C. Wallace, Proc. R. Soc. London. A 433 (1991) 631 